\title{
Effects of different surface finishing protocols for zirconia on surface roughness and bacterial biofilm formation
}

\author{
Du-Hyeong Lee', Hang-Nga Mai ${ }^{1}$, Phyu Pwint Thant ${ }^{1}$, Su-Hyung Hong' ${ }^{2}$ Jaewon Kim ${ }^{3}$, \\ Seung-Mi Jeong ${ }^{4}$, Keun-Woo Lee ${ }^{5 *}$ \\ 'Department of Prosthodontics, School of Dentistry, ITRD, Kyungpook National University, Daegu, Republic of Korea \\ ${ }^{2}$ Department of Microbiology and Immunology, School of Dentistry, Kyungpook National University, Daegu, Republic of Korea \\ ${ }^{3}$ Department of Periodontics and Endodontics, State University of New York at Buffalo, Buffalo, New York, United states of America \\ ${ }^{4}$ Department of Dentistry, Yonsei University Wonju College of Medicine, Wonju, Republic of Korea \\ ${ }^{5}$ Department of Prosthodontics, College of Dentistry, Yonsei University, Seoul, Republic of Korea
}

\begin{abstract}
PURPOSE. Surface finishing of a zirconia restoration is essential after clinical adjustment. Herein, we investigated the effects of a surface finishing protocol for monolithic zirconia on final roughness and bacterial adherence. MATERIALS AND METHODS. Forty-eight disk-shaped monolithic zirconia specimens were fabricated and divided into four groups $(n=12)$ based on initial surface treatment, finishing, and polishing protocols: diamond bur+polishing bur (DP group), diamond bur+stone grinding bur+polishing bur (DSP group), no diamond bur+polishing bur (NP group), and no diamond bur+stone grinding bur+polishing bur (NSP group). Initial and final surface roughness was measured with a profilometer, and shown using scanning electron microscope. Bacterial adhesion was evaluated by quantifying Streptococcus mutans in the biofilm. KruskalWallis and Mann-Whitney $U$ tests were used to compare results among groups, and two-way analysis of variance was used to evaluate the effects of grinding burs on final roughness $(\alpha=.05)$. RESULTS. The DP group had the highest final Ra value, followed by the DSP, NP, and NSP groups. Use of the stone grinding bur as a coarsefinishing step significantly decreased final Ra values when a diamond bur was used $(P<.001)$. Omission of the stone grinding bur increased biofilm formation on specimen surfaces. Combining a stone grinding bur with silicone polishing burs produced the smallest final biofilm values, regardless of the use of a diamond bur in initial surface treatment. CONCLUSION. Coarse finishing of monolithic zirconia with a stone grinding bur significantly decreased final Ra values and bacterial biofilm formation when surfaces had been roughened by a diamond bur. [J Adv Prosthodont 2019;11:41-7]
\end{abstract}

KEYWORDS: Zirconia; Dental finishing; Dental polishing; Biofilm; Bacterial adhesion

Corresponding author:

Keun-Woo Lee

Department of Prosthodontics, College of Dentistry, Yonsei University,

50 Yonsei-ro, Seodaemun-gu, Seoul 03722, Republic of Korea

Tel. +82222283158: e-mail, kwlee@yuhs.ac

Received July 10, 2018 / Last Revision December 24, 2018 / Accepted January 21, 2019

(C) 2019 The Korean Academy of Prosthodontics

This is an Open Access article distributed under the terms of the Creative Commons Attribution Non-Commercial License (http://creativecommons. org/licenses/by-nc/4.0) which permits unrestricted non-commercial use, distribution, and reproduction in any medium, provided the original work is properly cited.

This work was supported by the National Research Foundation of Korea (NRF grant funded by the Korea government (MSIP) (2017R1C1B2004976).

\section{INTRODUCTION}

Monolithic zirconia restorations are alternatives to layered zirconia restorations because of their decreased potential for chipping of the porcelain veneer. ${ }^{1}$ In a monolithic zirconia restoration, zirconia is directly exposed to the oral environment and must be polished following occlusal and axial adjustments. ${ }^{2}$ Surface smoothing of dental restorations is essential for reducing plaque accumulation, improving patient comfort, preventing wear of the antagonist enamel, and improving the aesthetics of restorations..$^{3-6}$ The effectiveness of a finishing and polishing system on dental material is evaluated by measuring the achieved surface roughness. ${ }^{2}$ Restorations with surface roughness values $>0.2 \mu \mathrm{m}$ 
(Ra) could trigger bacterial plaque accumulation, which is the primary cause of gingival inflammation and secondary caries. ${ }^{5}$ Bacteria adhere to restorations and natural teeth through a four-phase process. ${ }^{7,8}$ The initial phase begins with transport of bacteria toward the surface. In the second phase, bacteria adhere to the surface through a combination of Van der Waals forces and electrostatic repulsive forces. The intermediate attachment phase involves the adherence of bacteria to complementary sites on the substrate surface. In the final phase, bacteria colonize on the surface and form a biofilm via proliferation of already-colonized bacteria and/or by continuous adhesion of additional salivary bacteria. Initial adhesion of bacteria to the tooth surface is a critical step in bacterial plaque formation. ${ }^{9}$ The irregular geometry of a rough surface shelters bacteria from shear forces, giving them more time to interact with the surface and establish strong adhesion. ${ }^{7,9}$

Monolithic zirconia restoration fabricated from a solid block of zirconia has better mechanical properties and superior fracture strength than bi-layered restorations due to the uniform structure. ${ }^{10,11}$ The high strength and hardness of zirconia require specific surface treatment methods. ${ }^{1,12}$ Coarse-grit and fine-grit diamond rotary burs are often used at high speed for polishing monolithic zirconia ${ }^{13}$ because diamond burs are effective for extensive grinding of zirconia. Ho et $a l^{13}{ }^{13}$ stated that the use of diamond bur increased the flexural strength of zirconia, whereas Kosmac et al. ${ }^{14}$ concluded that use of the coarse diamond bur reduced the strength of the material due to surface flaws and microcracks. Therefore, specific zirconia finishing and polishing systems that incorporate instruments with improved diamond particle coatings have been developed. ${ }^{15}$ Use of zirconia-specific grinding burs are associated with higher grinding efficiency and lower heat generation than conventional stone burs. ${ }^{16}$

There have been reports in the literature on the importance of using specific stone burs on zirconia. ${ }^{16}$ However, the effects of stone burs in relation to use of different initial surface treatments on final roughness have not been fully clarified. The purpose of the present study was to examine whether use of diamond and stone burs on zirconia affects its final roughness and bacterial adhesion properties. The null hypothesis was that the surface grinding protocol for zirconia does not influence the final roughness or bacterial biofilm formation.

\section{MATERIALS AND METHODS}

Figure 1 presents the study design. Forty-eight disk-shaped monolithic zirconia specimens were designed using computer software (CATIA V5R19, Dassault Systemes, VelizyVillacoublay, France) and were manufactured with a 5-axis milling machine (Ceramill Motion 2, Amann Girrbach, Koblach, Austria) using zirconium dioxide blocks (Prettau, Zirkonzhan, Bruneck, Italy). Afterwards, sintering process of specimens was conducted using a dedicated sintering furnace (Zirkonofen 600/V2, Zirkonzahn). For the initial surface treatment, 24 specimens $(15 \mathrm{~mm}$ in diameter, $8 \mathrm{~mm}$ in thickness) were randomly selected and ground for $10 \mathrm{~s}$ under water-cooling conditions with a coarse-grit diamond

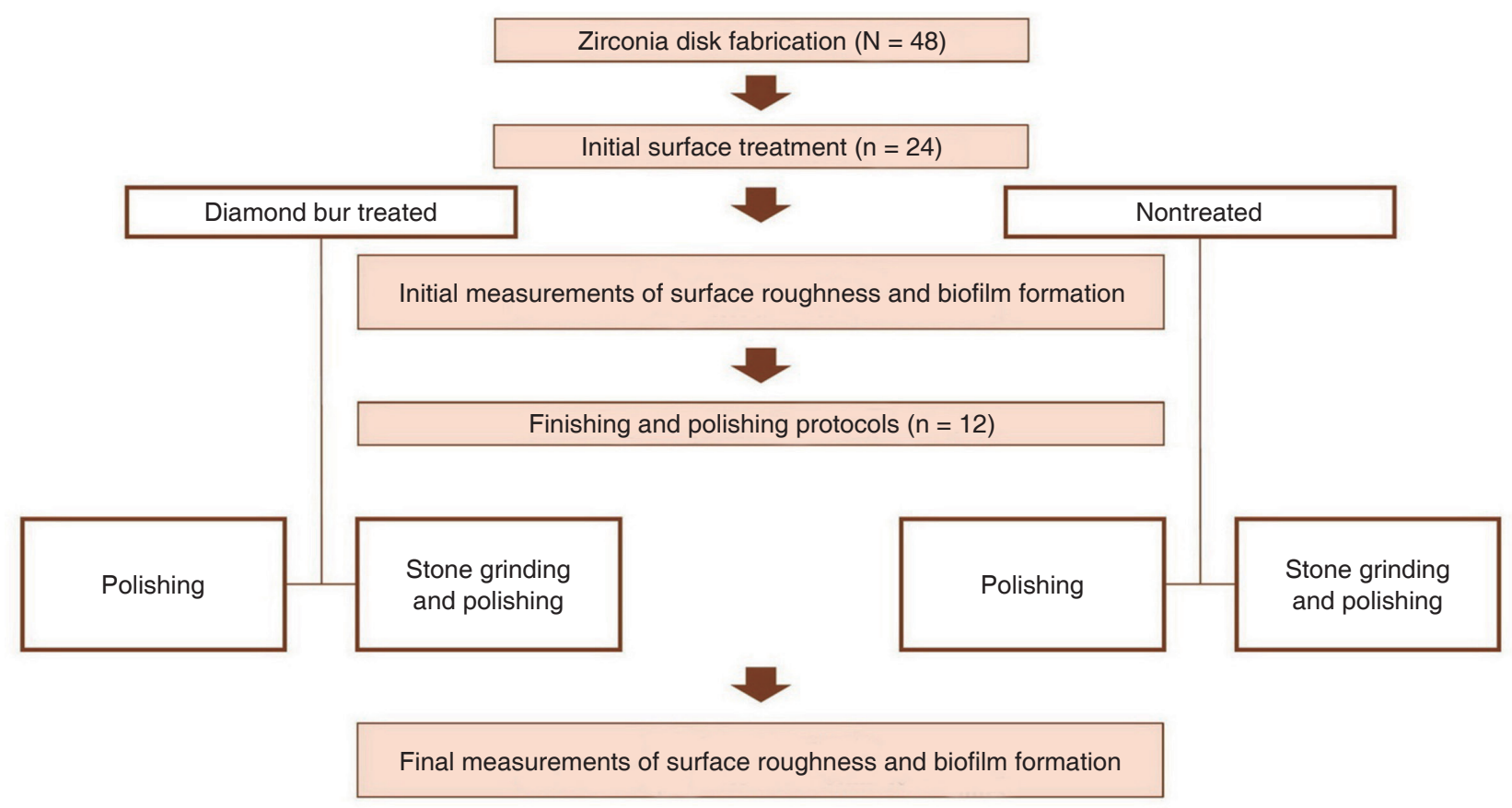

Fig. 1. Workflow of study. 
bur (V847KR, Edenta AG, Au, Switzerland) using an electronic high-speed handpiece (Ti-Max Z95L, NSK, Tokyo, Japan) to simulate the initial clinical adjustment. The surfaces of the remaining 24 specimens were not subjected to diamond bur adjustment. For the finishing step, half of the specimens in each group were ground with a stone bur. The specimens were then divided into four groups $(n=12)$, based on the initial treatment, finishing, and polishing protocols: diamond bur + polishing bur (DP group), diamond bur + stone grinding bur + polishing bur (DSP group), no diamond bur + polishing bur (NP group), no diamond bur + stone grinding bur + polishing bur (NSP group). After the coarse diamond bur grinding, finishing and polishing procedures, the specimens were cleaned by ultrasonication for 10 minutes in sterile distilled water, air blown, and stored dry at room temperature. All finishing and polishing procedures were performed by one operator using the Edenta system (Magic KIT Zir, Edenta GmbH, Lustenau, Austria) and a low-speed electronic handpiece (KaVo EXPERTmatic E10 C, KaVo, Biberach, Germany) with backward movement at 20 seconds per step, in accordance with the manufacturers' instructions. The instruments' codes and revolutions per minute used for surface treatments in this study are presented in Table 1. A microscope (MM 200, Nikon Corp., Tokyo, Japan) was used to observe the various sizes of natural diamond particles impregnated into instruments at $\times 300$ magnification (Fig. 2).
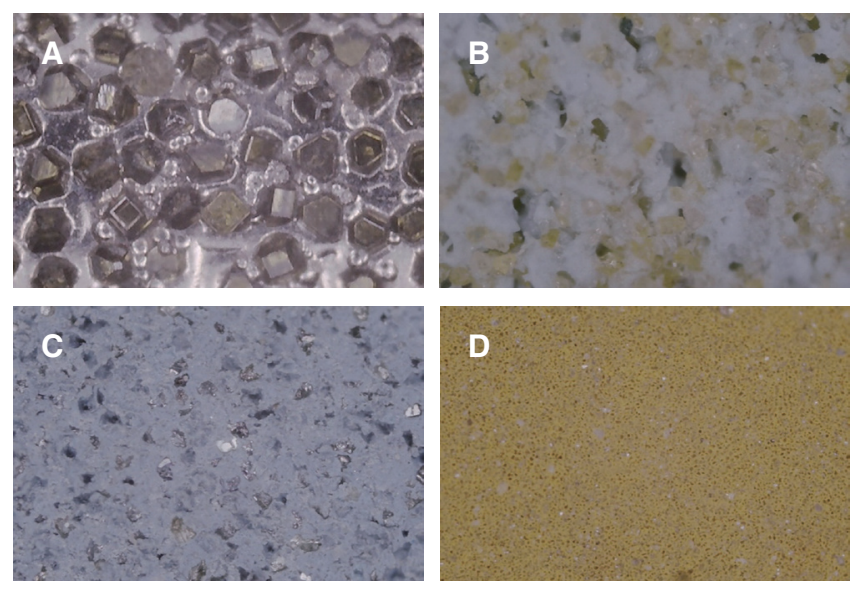

Fig. 2. Microscopic views of burs at $\times 300$ magnification. (A) Diamond bur, (B) Stone grinding bur, (C) Silicone polishing bur, (D) Fine silicone polishing bur.
The resulting surface roughness was measured with a contact surface profilometer (Surftest SV-400, Mitutoyo, Kawasaki, Japan) under a constant load of $4 \mathrm{mN}$ at a speed of $0.25 \mathrm{~mm} / \mathrm{s}$ and a measurement range of $0.8 \mathrm{~mm}$. The Ra value of each specimen was determined by averaging nine measurements: three repetitions of three traces.

Streptococcus mutans (ATCC 25175) was used to assess biofilm formation. $S$. mutans was maintained in a brain heart infusion (BHI) medium and grown under aerobic conditions. An in vitro biofilm formation assay was performed in accordance with published protocol. ${ }^{17}$ The specimens were cleaned with $70 \%$ alcohol and sterilized by UV light for 1 hour before the biofilm formation test. Briefly, S. mutans colonies were inoculated into BHI-1\% sucrose broth and incubated overnight. The culture broth was inoculated into $2 \mathrm{~mL}$ of the same liquid medium to reach $1 \%$ volume/volume in a 12 -well polystyrene plate containing each specimen. After incubation for 16 hours, the streptococcal broth was removed, and the specimens were washed three times with sterile phosphate buffered saline solution to remove loosely attached biomass. For biofilm quantification, $200 \mu \mathrm{L}$ of crystal violet solution $(0.2 \% \mathrm{w} / \mathrm{v}$ in $10 \%$ ethanol) was added to each specimen, followed by incubation for 1 hour. After washing with phosphate-buffered saline solution three times, the specimen was air-dried. Crystal violet retained by the streptococcal biofilm was redissolved in $200 \mu \mathrm{L}$ acidic solvent (10\% acetic acid in distilled water); the absorbance was determined with a microplate reader (Molecular Devices, San Jose, CA, USA) at $595 \mathrm{~nm} .^{18}$

For scanning electron microscope (SEM) analysis, the specimens were attached to an aluminum metal platform with a double sided adhesive tape. Then the edges of the specimens were painted with carbon paint (Pelco Colloidal Graphite, Ted Pella, Redding, CA, USA). After coating the specimen with spotted platinum with ion sputter (E-1030, Hitachi, Tokyo, Japan), they were analyzed with SEM (SU8230, Hitachi) at $\times 200$ magnification.

All statistical analyses were conducted using the IBM SPSS Statistics v22.0 statistical software package (IBM Corp., Armonk, NY, USA). Surface roughness for each group was expressed as mean \pm standard deviation. Kruskal-Wallis and Mann-Whitney U tests were used to compare the effects of stone grinding bur use among all subgroups. Two-way analysis of variance (ANOVA) was performed to investigate relationships between surface preparation and use of a stone grinding bur. Results were visualized using interaction plots. Statistical significance was set at $P<.05$.

Table 1. Instrument codes and revolutions per minute used for finishing and polishing

\begin{tabular}{lcccc}
\hline \multicolumn{1}{c}{ Category } & Diamond bur & Stone grinding bur & Silicone polishing bur & Fine silicone polishing bur \\
\hline Instrument codes* & V847KR & $8001.050 . \mathrm{HP}$ & $3041 . \mathrm{HP}$ & $30041 . \mathrm{HP}$ \\
Revolutions per minute & 200.000 & 12.500 & 20.000 & 10.000 \\
\hline
\end{tabular}

${ }^{*}$ As provided by manufacturers 


\section{RESULTS}

The initial surface roughness values were $\mathrm{Ra}=1.07 \mu \mathrm{m}$ for the diamond bur-treated group and $\mathrm{Ra}=0.42 \mu \mathrm{m}$ for the non-treated group. Table 2 presents the roughness of zirconia specimens by surface treatment. In the initial measurements, the use of the diamond bur at high speed significantly increased Ra values. After different finishing and polishing protocols, the DP group had the highest final Ra value, followed by the DSP, NP, and NSP groups. Addition of a finishing step using a stone grinding bur significantly decreased the final Ra values for diamond bur-treated specimens; however, the effect was not statistically significant when the specimens had not been treated with the diamond bur $(P=.70)$. Two-way ANOVA revealed a strong interaction between the use of the diamond bur in the initial surface treatment and the use of the stone grinding bur in the finishing step on the final $\mathrm{Ra}(P<.001)$ (Table 3$)$.

In vitro streptococcal biofilm masses on the surfaces of the specimens, as determined by crystal violet staining, were compared (Fig. 3). In general, biofilm formation of $S$. mutans significantly increased on the surfaces of specimens treated with the diamond bur, especially when use of a stone grinding bur was omitted $(P<.001)$. The combination of a stone grinding bur and a silicone polishing bur produced small final biofilm mass.

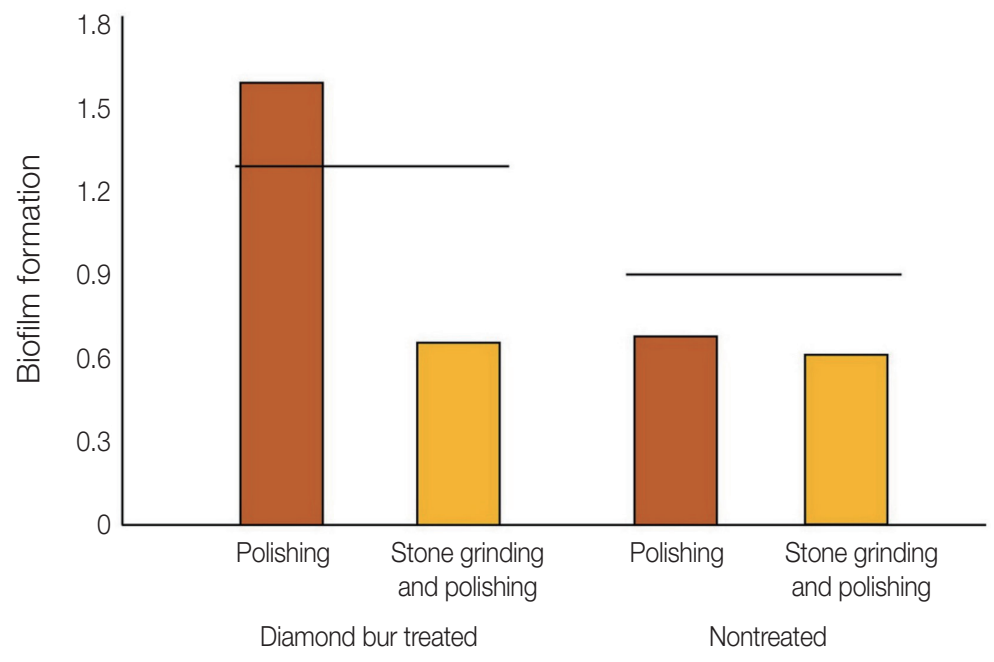

Fig. 3. Biofilm formation on zirconia specimen from each group after finishing and polishing. Black lines indicate initial biofilm formation for diamond bur-treated and nontreated specimens.

Table 2. Means \pm SDs for surface roughness (Ra) of specimens by surface treatment protocol

\begin{tabular}{cccccc}
\hline & DP & DSP & NP & NSP & $P$ \\
\hline Roughness $(\mu \mathrm{m})$ & $0.87 \pm 0.11^{\mathrm{a}}$ & $0.64 \pm 0.10^{\mathrm{b}}$ & $0.32 \pm 0.06^{\mathrm{c}}$ & $0.29 \pm 0.07^{\circ}$ & $<.001$ \\
\hline
\end{tabular}

DP: diamond bur + polishing bur, DSP: diamond bur + stone grinding bur + polishing bur, NP: no diamond bur + polishing bur, NSP: no diamond bur + stone grinding bur + polishing bur.

Significant differences are represented by different superscript lowercase letters within a row.

Table 3. Two-way ANOVA for surface roughness

\begin{tabular}{|c|c|c|c|c|c|}
\hline Source & Sum of squares & d.f. & Mean square & $\mathrm{F}$ & $P$ \\
\hline Diamond bur & 2.417 & 1 & 2.417 & 331.675 & $<.001$ \\
\hline Stone bur & .189 & 1 & .189 & 25.907 & $<.001$ \\
\hline Diamond bur $\times$ Stone bur & .121 & 1 & .121 & 16.608 & $<.001$ \\
\hline Error & .321 & 44 & .007 & & \\
\hline Total & 16.583 & 48 & & & \\
\hline Corrected total & 3.047 & 47 & & & \\
\hline
\end{tabular}



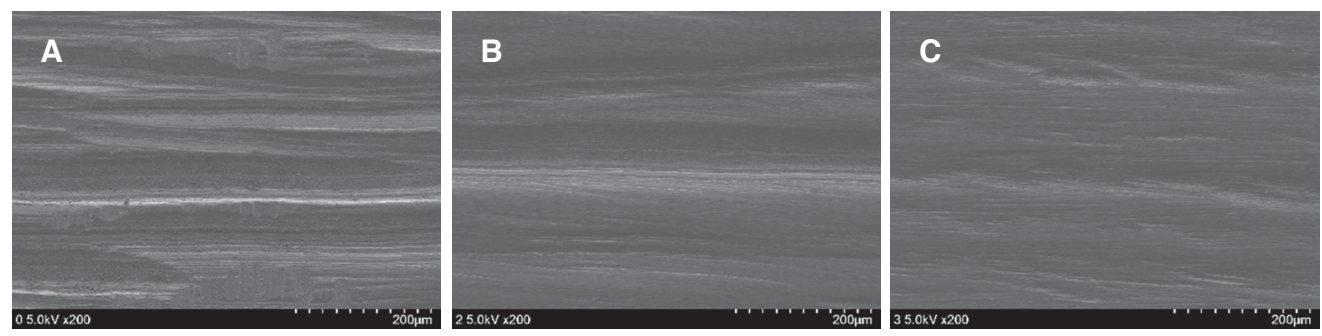

Fig. 4. Scanning electron microscope images of diamond bur-treated specimens. (A) Surface after use of diamond bur alone, (B) Surface after use of diamond and polishing burs, (C) Surface after use of diamond bur, stone grinding bur, and polishing bur.

For SEM images, the specimen treated with a diamond bur alone showed sharp groove patterns (Fig. 4A), as opposed to the other specimens (Fig. 4B, 4C). The specimen of DSP group showed more evenness of the surface compared to rounded edges of the large grooves of DP group (Fig. 4B, 4C).

\section{DISCUSSION}

This study was conducted to evaluate the effects of surface finishing of monolithic zirconia with a coarse stone grinding bur on final surface roughness and bacterial adhesion. Based on our results, the null hypothesis was rejected because the final surface roughness and biofilm formation were significantly different, depending on the coarse-finishing protocols used.

A zirconia-specific stone grinding bur was used in this study in a coarse-finishing step for specimens with different initial surface roughness values. When initial surface treatment is performed using a high-speed diamond bur on monolithic zirconia specimens, a very rough surface is produced. The finishing and polishing procedure is used to achieve a smooth and regular surface for the restoration. However, deep scratches created by the diamond bur are difficult to remove with silicone polishing burs because of the extreme strength and hardness of zirconia material. Finishing and polishing instruments comprise of three major components: primary abrasive, supplemental abrasive, and abrasive-fixing substance. Among these, the primary abrasive is the main factor determining the grinding efficiency. ${ }^{11}$ Since zirconia has a value of 9 on the Mohs hardness scale, diamond grinding burs for occlusal adjustment have been manufactured using a coarse diamond grain size and serve as the primary abrasive. ${ }^{16}$ Silicone polishing burs, on the other hand, contain very fine diamond grains; thus, these burs exhibit significantly lower grinding efficiency. Therefore, use of a stone grinding bur may be considered an important intermediate step in filling the gap between the extremely rough surface of the diamond bur and the fine surfaces of silicone polishing burs. Previous studies have reported that use of zirconia stone grinding burs for zirconia materials plays an important role in removing deep grooves and achieving a finer surface texture for subsequent polishing steps. ${ }^{16}$ Therefore, addition of a coarse-finishing step using a stone grinding bur is essential and should not be omitted in clinical practice, particularly after extensive reduction of the zirconia restoration is accomplished with a coarse-grit diamond bur.

Surface roughness is considered the most important factor in oral biofilm formation. ${ }^{9}$ A correlation between surface roughness and bacterial adhesion was also observed in this study. Biofilm formation was substantially reduced after stone grinding, independent of whether specimens had been roughed by diamond burs. Interestingly, when use of the stone grinding bur was omitted, use of silicone polishing burs alone increased the biofilm thickness in specimens treated with a diamond bur. This phenomenon may be explained by the surface topography of treated zirconia (Fig. 4, Fig. 5). The initial surface treatment process that used a coarse-grit diamond bur generated deep, sharp grooves on the surface. The silicone bur is not suitable for grinding irregular macrostructures because of the low hardness and fine grit of the diamond particles in the instrument. Moreover, silicone burs cannot reach the inner sides of the narrow deep grooves, but they can be used to round the sharp outer edges of these grooves. Although this partial surface alteration generally decreases the Ra value of polished zirconia, this surface structure may provide more favorable conditions for bacterial adhesion. Han et al. ${ }^{19}$ also reported the similar bactericidal effects in the surface with sharp grooves and pits. These results confirm the clinical importance of sequential finishing and polishing regimens.

There are various clinical and technical factors that determine the final roughness of zirconia. The roles of various polishing systems and sintering processes in the final roughness of zirconia need to be evaluated in future studies. ${ }^{20,21}$ Moreover, the effects of grinding direction, time and pressure, and use of water coolants are also important clinical factors that require further investigation. 


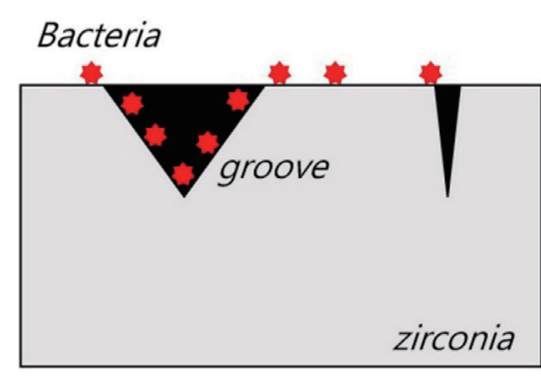

A

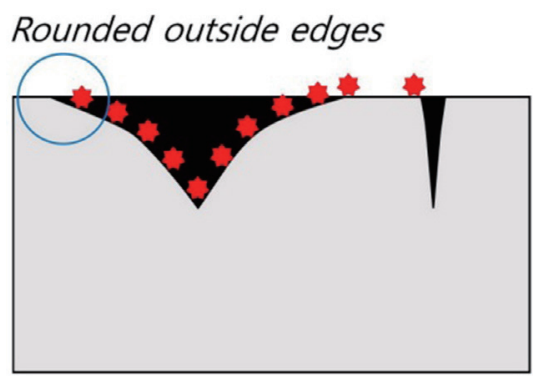

B

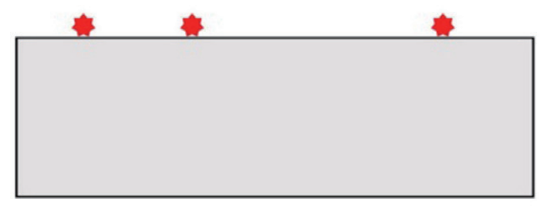

C

Fig. 5. Schematic image of bacterial adhesion to zirconia; heavy bacterial inhabitation is observed in the partially altered surface treated by diamond and polishing burs. (A) Surface after use of diamond bur alone, (B) Surface after use of diamond and polishing burs, (C) Surface after use of diamond bur, stone grinding bur, and polishing bur.

\section{CONCLUSION}

Within the limitations of this study, a coarse-finishing step using a stone grinding bur significantly decreased the final Ra values of monolithic zirconia when the surface had been roughed by a diamond bur. Biofilm formation of $S$. mutans increased on the surfaces of specimens treated with a diamond bur, especially when use of a stone grinding bur was omitted.

\section{ORCID}

Du-Hyeong Lee https://orcid.org/0000-0003-2803-7457

Hang-Nga Mai https://orcid.org/0000-0002-9832-3312

Phyu Pwint Thant bttps://orcid.org/0000-0002-7326-2971

Su-Hyung Hong https://orcid.org/0000-0001-5569-3826

Jaewon Kim https://orcid.org/0000-0001-7952-0815

Seung-Mi Jeong https://orcid.org/0000-0002-1543-7227

Keun-Woo Lee https://orcid.org/0000-0002-3153-190X

\section{REFERENCES}

1. Caglar I, Ates SM, Yesil Duymus Z. The effect of various polishing systems on surface roughness and phase transformation of monolithic zirconia. J Adv Prosthodont 2018;10: 132-7.

2. Chavali R, Lin CP, Lawson NC. Evaluation of different polishing systems and speeds for dental zirconia. J Prosthodont 2017;26:410-8.

3. Kim HK, Kim SH, Lee JB, Ha SR. Effects of surface treatments on the translucency, opalescence, and surface texture of dental monolithic zirconia ceramics. J Prosthet Dent 2016; 115:773-9.

4. Kim MJ, Oh SH, Kim JH, Ju SW, Seo DG, Jun SH, Ahn JS, Ryu JJ. Wear evaluation of the human enamel opposing different Y-TZP dental ceramics and other porcelains. J Dent 2012;40:979-88.

5. Teughels W, Van Assche N, Sliepen I, Quirynen M. Effect of material characteristics and/or surface topography on biofilm development. Clin Oral Implants Res 2006;17:68-81.

6. Jones CS, Billington RW, Pearson GJ. The in vivo perception of roughness of restorations. Br Dent J 2004;196:42-5.

7. Yuzugullu B, Celik C, Burak Ozcelik T, Erkut S, Yurdakul P, Ocal Y, Sener B. The effect of different polishing sequences on the adhesion of Streptococcus mutans to feldspathic Porcelain. J Adhes 2016;92:939-49.

8. Bollen CM, Lambrechts P, Quirynen M. Comparison of surface roughness of oral hard materials to the threshold surface roughness for bacterial plaque retention: a review of the literature. Dent Mater 1997;13:258-69.

9. Lee BC, Jung GY, Kim DJ, Han JS. Initial bacterial adhesion on resin, titanium and zirconia in vitro. $\mathrm{J} \mathrm{Adv}$ Prosthodont 2011;3:81-4.

10. Miyazaki T, Nakamura T, Matsumura H, Ban S, Kobayashi T. Current status of zirconia restoration. J Prosthodont Res 2013;57:236-61.

11. Huh YH, Park CJ, Cho LR. Evaluation of various polishing systems and the phase transformation of monolithic zirconia. J Prosthet Dent 2016;116:440-9.

12. Preis V, Grumser K, Schneider-Feyrer S, Behr M, Rosentritt M. The effectiveness of polishing kits: influence on surface roughness of zirconia. Int J Prosthodont 2015;28:149-51.

13. Ho CM, Ding H, Chen X, Tsoi JK, Botelho MG. The effects of dry and wet grinding on the strength of dental zirconia. Ceram Int 2018;44:10451-62.

14. Kosmac T, Oblak C, Jevnikar P, Funduk N, Marion L. Strength and reliability of surface treated Y-TZP dental ceramics. J Biomed Mater Res 2000;53:304-13.

15. Kosmac T, Oblak C, Jevnikar P, Funduk N, Marion L. The effect of surface grinding and sandblasting on flexural strength and reliability of Y-TZP zirconia ceramic. Dent Mater 1999; 15:426-33.

16. Lee KR, Choe HC, Heo YR, Lee JJ, Son MK. Effect of different grinding burs on the physical properties of zirconia. J Adv Prosthodont 2016;8:137-43. 
17. Gilan I, Sivan A. Extracellular DNA plays an important structural role in the biofilm of the plastic degrading actinomycete Rhodo-coccus ruber. Adv Microbiol 2013;3:543-51.

18. O’Toole GA. Microtiter dish biofilm formation assay. J Vis $\operatorname{Exp} 2011 ; 47: 2437$.

19. Han A, Tsoi JKH, Matinlinna JP, Chen Z. Influence of gritBlasting and hydrofluoric acid etching treatment on surface characteristics and biofilm formation on zirconia. Coatings 2017;7:130.

20. Kim HK, Kim SH. Comparison of the optical properties of pre-colored dental monolithic zirconia ceramics sintered in a conventional furnace versus a microwave oven. J Adv Prosthodont 2017;9:394-401.

21. Goo CL, Yap A, Tan K, Fawzy AS. Effect of polishing systems on surface roughness and topography of monolithic zirconia. Oper Dent 2016;41:417-23. 